\title{
Common Mode Characterization and Channel Model Verification for Shielded Twisted Pair (STP) Cable
}

\author{
Miloš Jakovljević*, Thomas Magesacher ${ }^{\dagger \ddagger}$, Klas Ericson ${ }^{\S}$, Per Ödling ${ }^{\dagger}$, Per Ola Börjesson ${ }^{\dagger}$, and Santiago Zazo* \\ ${ }^{*}$ ETSIT-SSR, Signal Processing Application Group (GAPS), Ciudad Universitaria s/n, 28040 Madrid, Spain \\ email: \{milos, santiago\}@gaps.ssr.upm.es \\ $\dagger$ Department of Information Technology, Lund University, P.O. Box 118, S-22100 Lund, Sweden \\ email: \{tom, per, pob\}@eit.lth.se \\ ${ }^{\ddagger}$ Department of Electrical Engineering, Stanford University, CA 94305-9515, USA

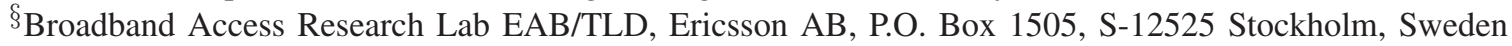 \\ email: klas.ericson@ericsson.com
}

\begin{abstract}
This paper investigates common-mode propagation in shielded twisted pair cables. The common mode exhibits great potential for improving the throughput in emerging wireline systems. The design of corresponding transmission schemes over multipair copper cables requires accurate knowledge of the channel properties. We present measurement and modeling results and investigate the feasibility of using standard differentialmode models for data fitting in multiconductor transmission-line modelling of common-mode paths.
\end{abstract}

\section{INTRODUCTION}

During the last two decades, several families of digital subscriber line (DSL) have been developed, standardized, and deployed. The major impairments to DSL performance are loop attenuation and crosstalk. Loop attenuation increases with both loop length and frequency. At both ends of any pair in a cable, remnants of the signals transmitted in neighboring pairs are detectable. In case the crosstalk source is located at the opposite end as the receiver, the unwanted effect is referred as far-end crosstalk (FEXT). FEXT often limits the achievable datarate in high-throughput systems.

The differential mode (DM), conventionally used in today's systems, is the only propagation mode in a two conductor system. Signals are transmitted as voltage differences between the two wires of a twisted pair. Well established DM channel models are available (see, for example, [1] and references therein). Augmenting a two-wire system by earth (or local common), treated as a third conductor, yields a second independent mode, the so-called common mode (CM). Recently, interest for CM aided DSL-transmission emerged. Treating the $\mathrm{CM}$ signal as an additional receive signal, the capacity of the resulting channel for different levels of coordination among the users was investigated in [2]. To determine the possible gain, cable measurement results of crosstalk channels using $\mathrm{CM}$ mode are used.

Next-generation techniques treat the FEXT-impaired twisted-pair binder as a multiple-input multiple-output (MIMO) channel. DM MIMO measurements are presented in [3]. In [4], the DM model from [5] was verified through measurements. In [6], promising results using vectoring [7] in combination with $\mathrm{CM}$ transmission are reported. A corresponding CM MIMO channel model based on multiconductor transmission-line theory [8] is introduced in [5], [9]. One of the wires in the cable is used as a common reference. Clearly, the CM is likely to experience higher extrinsic noise levels. Furthermore, electromagnetic compatibility requirements may impose restrictions on the admissible transmit power spectral densities. Nevertheless, the number of modes in such a CM configuration almost doubles compared to the DM configuration.

This paper focuses on shielded twisted pair (STP) cables and investigates $\mathrm{CM}$ propagation in the configuration proposed in [5], [9], however, with the shield used as common reference. The number of modes in such a configuration doubles compared to the DM configuration. More importantly, the shield substantially reduces the level of extrinsic noise entering the cable and relaxes the electromagnetic compatibility issue. Considering these aspects, exploiting the CM in STP cables may have great potential. The paper is organized as follows: Section II briefly reviews the reference models from [5] and [9] together with adaptations for STP cables. Section III describes the measurement setup used to obtain data for parametrization and verification. Section IV presents measurement and modelling results and Section V concludes the paper.

\section{REFERENCE MODELS}

\section{A. Multiconductor Transmission-Line Modelling}

In order to apply multiconductor transmission-line theory, a cable of length $L$ with $m$ pairs is treated as a cascade of many short segments represented as lumped equivalent circuits whose parameters are per length-unit quantities [9]. These segments can be characterized by the matrix parameters $\boldsymbol{R}$, $L, C$, and $G$. In case one of the wires is used as a reference conductor, the size of the matrices is $(2 m-1) \times(2 m-1)$, like in [9]. When a cable has a shield, [8] suggests that the 
shield can be modeled as an additional conductor. Thus, there are $2 m+1$ conductors and the general theory presented in [9] still applies, but the matrices have size $2 m \times 2 m$. Therefore, for STP cables, the off-diagonal elements of the matrix $\boldsymbol{R}$ are given by the per length-unit resistance $r_{0}$ of the shield. The $j$ th diagonal element of the matrix $\boldsymbol{R}$ is given by the sum of the per length-unit resistance of conductor No. $j$ and the per length-unit resistance $r_{0}$ of the shield. The diagonal entry $l_{j j}$ of the matrix $\boldsymbol{L}$ is given by the per length-unit inductance of conductor No. $j$ toward the shield. The off-diagonal element $l_{i j}, i \neq j$ is given by the per length-unit mutual inductance between conductor No. $i$ and conductor No. $j$. Analogously, the elements of the matrices $C$ and $G$ represent per length-unit capacitance and conductance (toward the shield or between conductors No. $i$ and No. $j$ ), respectively. Note that all matrix entries depend on the frequency $f$.

On each side of the cable, $2 m$ voltages $V_{j}, 1 \leq j \leq 2 m$ and $2 m$ currents $I_{j}, 1 \leq j \leq 2 m$ can be defined. The

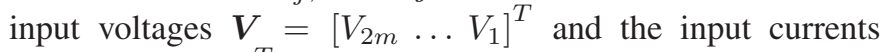
$\boldsymbol{I}=\left[I_{2 m} \ldots I_{1}\right]^{T}$ fulfill $\boldsymbol{V}=\boldsymbol{Z}_{\text {in }} \boldsymbol{I}$, where $\boldsymbol{Z}_{\text {in }}$ is the input impedance matrix. Denoting the termination admittance matrix by $\boldsymbol{Y}_{t}, \boldsymbol{V}^{\prime}=\boldsymbol{Y}_{t}^{-1} \boldsymbol{I}^{\prime}$ holds, where $\boldsymbol{V}^{\prime}=\left[V_{2 m}^{\prime} \ldots V_{1}^{\prime}\right]^{T}$ and $\boldsymbol{I}^{\prime}=\left[I_{2 m}^{\prime} \ldots I_{1}^{\prime}\right]^{T}$ are the output voltages and currents, respectively. It was demonstrated in [10] that the capacity when terminating the cable in $\boldsymbol{Y}_{t}=\operatorname{diag}\left(1 / Z_{C} \ldots 1 / Z_{C}\right)$ is the same as terminating the cable in $\boldsymbol{Y}_{t}=\boldsymbol{Y}_{C}$. Thus, we consider a diagonal termination admittance, which has more practical relevance.

The voltages $\boldsymbol{V}$ and $\boldsymbol{V}^{\prime}$ are related through the transfer function matrix ${ }^{1}$ given by

$$
\boldsymbol{T}=\left(\cosh \left(\boldsymbol{\gamma}^{T} L\right)+\sinh \left(\boldsymbol{\gamma}^{T} L\right) \boldsymbol{Y}_{t} \boldsymbol{Z}_{C}\right)^{-1}
$$

where $Z_{C}$ is the characteristic impedance matrix and $\gamma$ is its propagation matrix. The characteristic impedance matrix and the propagation matrix are given by

$$
Z_{C}=\sqrt{Z Y^{-1}}
$$

and

$$
\gamma=\sqrt{Z Y}
$$

respectively, where

$$
\boldsymbol{Z}=\boldsymbol{R}+j \omega \boldsymbol{L}
$$

is the symmetric per length-unit impedance matrix and

$$
\boldsymbol{Y}=\boldsymbol{G}+j \omega \boldsymbol{C}
$$

is the symmetric per length-unit admittance matrix. The explicit matrix forms for $\boldsymbol{R}, \boldsymbol{L}, \boldsymbol{C}$, and $\boldsymbol{G}$ are given in [9].

\section{B. Primary Parameter Modelling}

In this paper, we attempt to use the parametrized models presented in [5] for the elements of the matrices $\boldsymbol{R}, \boldsymbol{L}$, and $\boldsymbol{C}$. The conductance is neglected, as suggested in [5]. The model parameters are determined through non-linear least-square

\footnotetext{
${ }^{1}$ Note that all operators with matrix arguments denote matrix functions.
}

fitting of measurement data. The model for the resistance is given by

$$
r(f)=\frac{1}{2}\left(\frac{1}{\frac{1}{\left(r_{o c}^{4}+a_{c} \cdot f^{2}\right)^{\frac{1}{4}}}+\frac{1}{\left(r_{o s}^{4}+a_{s} \cdot f^{2}\right)^{\frac{1}{4}}}}\right),
$$

where $r_{o c}$ is the copper DC resistance and $r_{o s}$ is the steel DC resistance, while $a_{c}$ and $a_{s}$ characterize the rise of resistance with frequency due to the 'skin effect'. The model for inductance is given by

$$
l(f)=\frac{l_{(0)}+l_{(\infty)}\left(\frac{f}{f_{m}}\right)^{b}}{1+\left(\frac{f}{f_{m}}\right)^{b}},
$$

where $l_{(0)}$ and $l_{(\infty)}$ are the low-frequency inductance and high-frequency inductance, respectively, and $b$ is a parameter chosen to characterize the transition from low to high frequencies. Finally, the model for the capacitance is given by

$$
c(f)=c_{(\infty)}+c_{(0)} f^{-c_{(e)}},
$$

where $c_{(\infty)}$ is the 'contact' capacitance and $c_{(0)}$ and $c_{(e)}$ are additional fitting parameters.

\section{iII. Measurement Setup}

All measurements were performed on a shielded $0.5 \mathrm{~mm}$ cable (24AWG) with 10 pairs (vendor identification: ECAFK/100 10X2X0.5) of length $160 \mathrm{~m}$ coiled up on a drum. All impedance measurements were carried out with an impedance analyzer, model HP 4192A, in the frequency range $5 \mathrm{~Hz}-13 \mathrm{MHz}$. All transfer functions and coupling functions were measured with a gain/phase analyzer, model Agilent $4395 \mathrm{~A}$, for 801 frequencies in the range $3 \mathrm{kHz}-30 \mathrm{MHz}$.

\section{A. Parameter Measurements}

In order to use the model introduced in Section II, the matrices $R, L$, and $C$ need to be filled with values obtained through fitting measurement data.

a) Resistance $\boldsymbol{R}$ : The data for the diagonal elements of $\boldsymbol{R}$ was gathered by measuring secondary parameters (characteristic impedance and propagation constant) between each wire and the shield. For determining the secondary parameters, we follow a standard procedure based on measuring the input impedances $Z_{O C}$ and $Z_{S C}$ with open-circuit and shortcircuit far-end ports, respectively (see, for example, [3]). The primary parameters can be derived directly from the secondary parameters. According to [8], the off-diagonal elements of $\boldsymbol{R}$ can be assumed equal to the DC shield resistance per unit length.

b) Inductance $\boldsymbol{L}$ : The diagonal elements $l_{i i}$ of $\boldsymbol{L}$ are obtained from the secondary parameters of the common mode formed by conductor No. $i$ and the shield as described above. The off-diagonal elements $l_{i j}$, which are the mutual impedances between conductor No. $i$ and conductor No. $j$, are given by

$$
l_{i j}=\frac{1}{2}\left(l_{i i}+l_{j j}-l_{i j m}\right),
$$


where $l_{i i}$ is the inductance between conductor No. $i$ and the shield and $l_{i j m}$ is the measured inductance between conductor No. $i$ and conductor No. $j$. All other wires were left open on both ends as suggested in [11], [12].

c) Capacitance $C$ : According to [11], [12], the mutual capacitance $c_{i j}$ between conductor No. $i$ and conductor No. $j$ can be calculated as

$$
c_{i j}=\frac{c_{i}+c_{j}}{2}-\frac{c_{i j m}}{4},
$$

where $c_{i}$ is the capacitance measured between conductor No. $i$ and conductor No. $j$ when the far end of conductor $j$ is connected to all other wires and to the shield. Analogously, $c_{j}$ is the capacitance measured between conductor No. $i$ and conductor No. $j$ when the far end of conductor $i$ is connected to all other wires and to the shield. The capacitance $c_{i j m}$ is measured between the conductor No. $i$ and conductor No. $j$ with their far end short-circuited, and all far ends of all other conductors connected to the shield. Details regarding the measurement procedure are described in [11], [12]. The diagonal elements can be extracted by solving an equation system as described in [13].

\section{B. Frequency Response Measurements}

In an attempt to assess the quality of the models parametrized by the measured data, we compare commonmode paths derived from the model with direct measurements. We focus on insertion loss and FEXT coupling functions. For FEXT, we distinguish between crosstalk among wire-shield modes that share the same twisted pair and wire-shield modes in different pairs. All unused ports were terminated in $70 \Omega$.

\section{Measurement Results}

\section{A. Parameters Measurements}

Figure 1 shows the characteristic impedance obtained through open/short-circuit measurements both in differentialmode and in common-mode configuration. The characteristic impedance in differential-mode configuration is around $100 \Omega$ for high frequencies. The characteristic impedance in common-mode configuration is around $70 \Omega$.

Figure 2 shows attenuation $\alpha$ and phase $\beta$ of the propagation constant for both differential-mode and common-mode configuration. For high frequencies, the attenuation constant of the common mode is slightly higher compared to the differential mode, which results in a slightly larger insertion loss in common-mode configuration.

Figure 3 shows an exemplary per length-unit resistance obtained from the secondary parameters. The result measured between a conductor (for example No. $j$ ) and the shield is used as diagonal entry $r_{j j}$ in $\boldsymbol{R}$. For comparison, the resistance measured between two conductors that form a pair is shown as well. We attempt to fit the measured resistance using the model (1). Neither the fitting results nor the extrapolation beyond $13 \mathrm{MHz}$ are entirely convincing. Nevertheless, due to the lack of a better model, we proceed using (1).

Figure 4 shows an exemplary result of the per lengthunit inductance computed from the secondary parameters. The
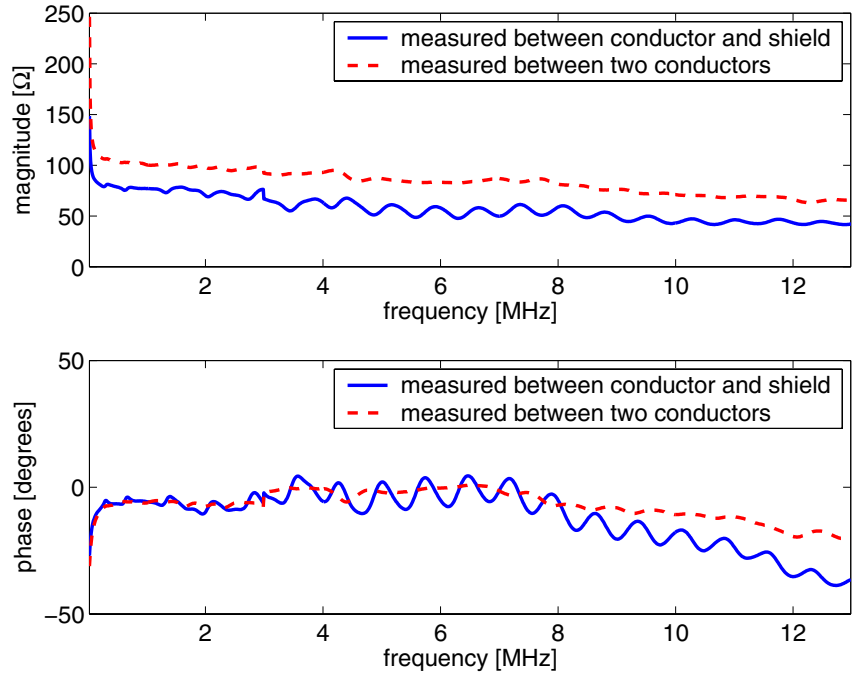

Fig. 1. Characteristic impedance measured between a conductor and the shield (solid line) and between two conductors (dashed line). Top plot: magnitude. Bottom plot: phase.
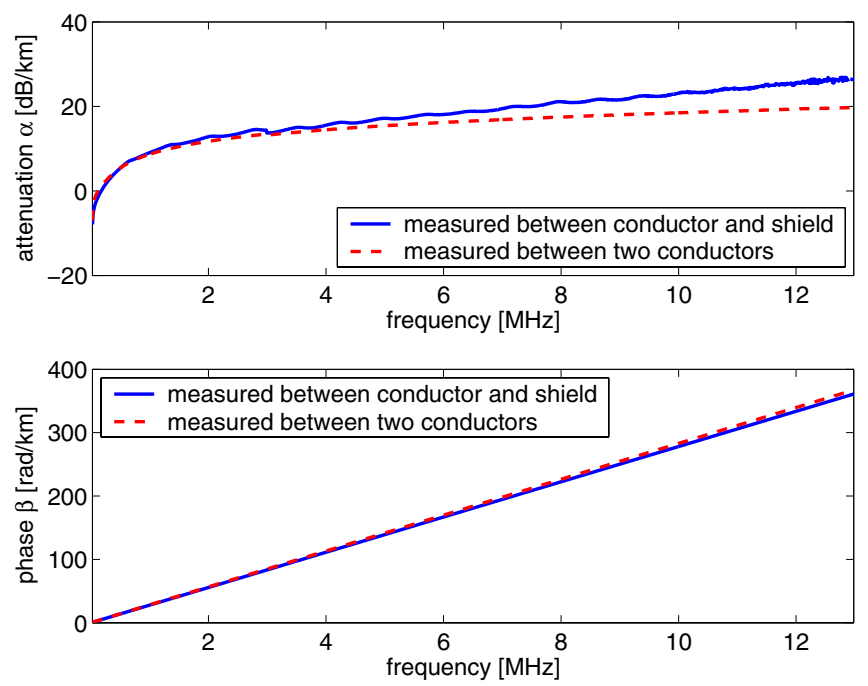

Fig. 2. Propagation constant measured between a conductor and the shield (solid line) and between two conductors (dashed line). Top plot: attenuation. Bottom plot: phase.

result measured between a conductor (for example No. $j$ ) and the shield is used both as diagonal entry $l_{j j}$ in $\boldsymbol{L}$ and to obtain the off-diagonal elements of $\boldsymbol{L}$ using (4). The result measured between two conductors (for example No. $i$ and No. $j$ ), which is used as variable $l_{i j m}$ in (4), is shown as well. Data fitting using the model (2) yields fairly good results. Extrapolation beyond $13 \mathrm{MHz}$ seems plausible (although, of course, we have no means of verification without measurements beyond $13 \mathrm{MHz}$ ).

Figure 5 shows exemplary results of the per length-unit capacitance. Both $c_{j}$ and $c_{i j m}$, which yield the element $c_{i j}$ of $C$ according to (5), are shown together with fitted and extrapolated values. Data fitting using the model (3) yields fairly good results. Extrapolation beyond $13 \mathrm{MHz}$ seems plausible 


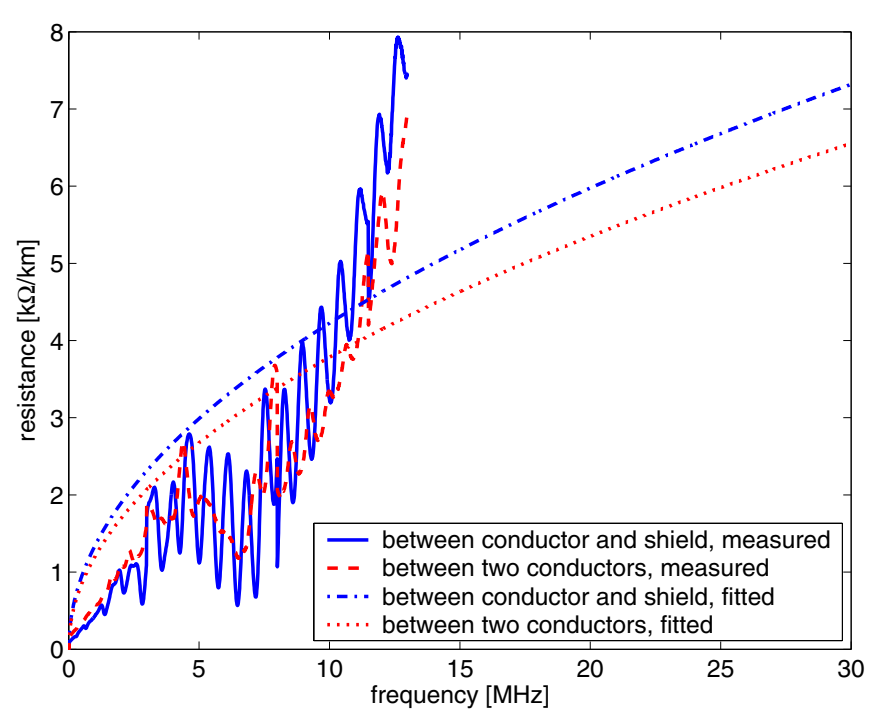

Fig. 3. Per length-unit resistance derived from secondary parameters. Resistance between one conductor and the shield (solid line: measured. dashed-dotted line: fitted) and between two conductors (dashed line: measured. dotted line: fitted).

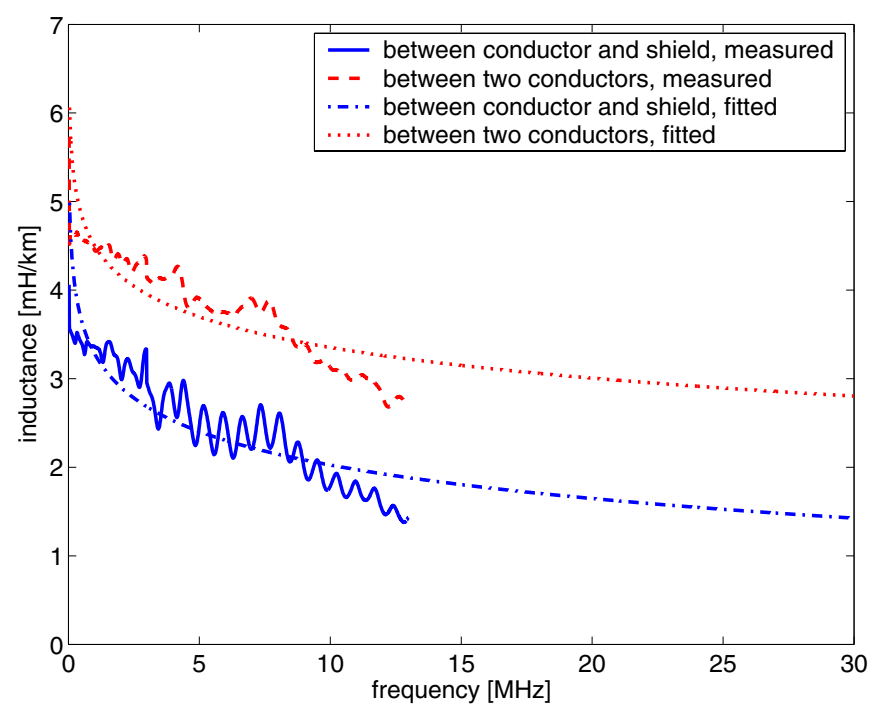

Fig. 4. Per length-unit inductance derived from secondary parameters. Inductance between one conductor and the shield (solid line: measured. dashed-dotted line: fitted) and between two conductors (dashed line: measured. dotted line: fitted).

(again, of course, we have no means of verification without measurements beyond $13 \mathrm{MHz}$ ).

\section{B. Frequency Response Measurements}

Figure 6 depicts the insertion loss of the common mode. Direct frequency-domain measurements of all 20 paths are shown together with frequency responses derived from parametrized models. As parameters we use the results of the data fitting. The match between the model with fitted parameters and

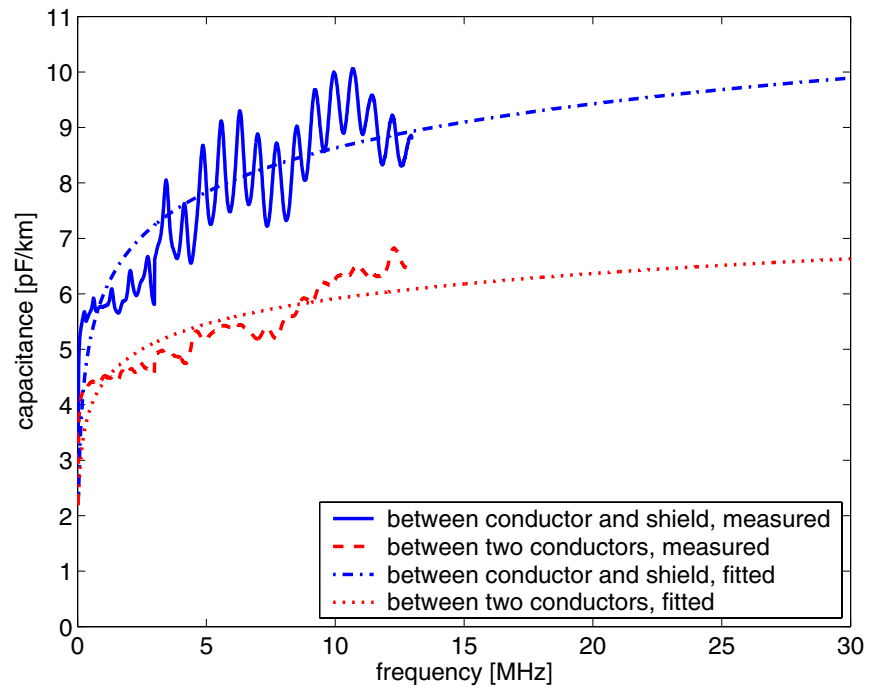

Fig. 5. Per length-unit capacitance obtained following procedure from [11], [12]. Measured capacitance between one conductor and the shield (solid line: measured. dashed-dotted line: fitted) and between two conductors (dashed line: measured. dotted line: fitted).
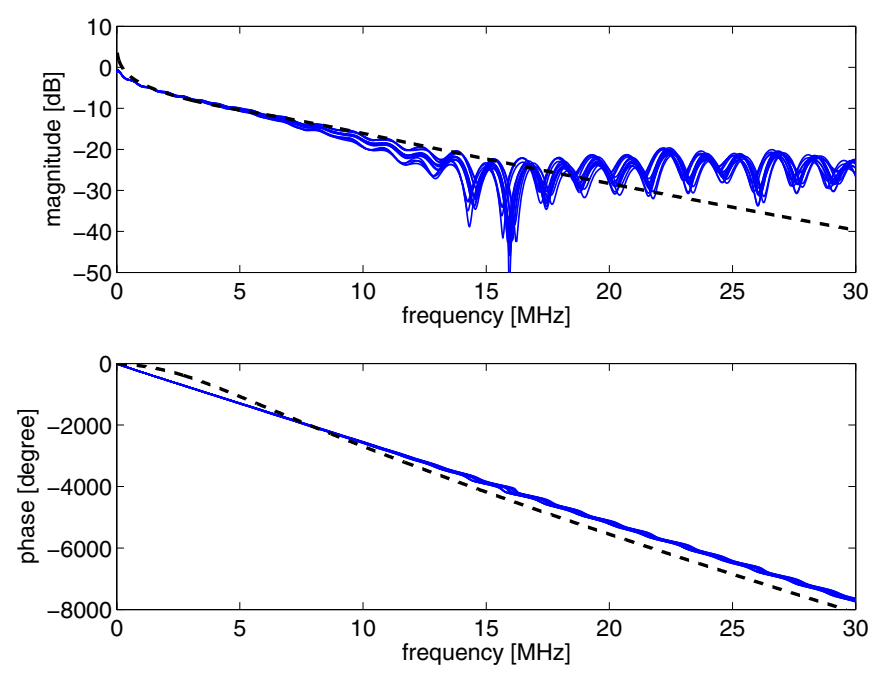

Fig. 6. Insertion loss of wire-shield modes (Top plot: magnitude. Bottom plot: phase). Solid lines: 20 measured wire-shield paths. Dashed line: model based on parameters obtained via data fitting.

directly measured results is reasonable except for frequencies beyond $13 \mathrm{MHz}$, where the data has been extrapolated.

Figures 7 and 8 show measured FEXT coupling functions between wire-shield modes whose wires belong to the same twisted pair (20 different measurements) and between wireshield modes whose wires belong to different pairs (360 different measurements), respectively. The presented modelling results are obtained with fitted data. The magnitude of the FEXT coupling observed among wire-shield modes whose wires belong to the same twisted pair is around $10 \mathrm{~dB}$ higher compared to the FEXT among wire-shield modes whose wires 

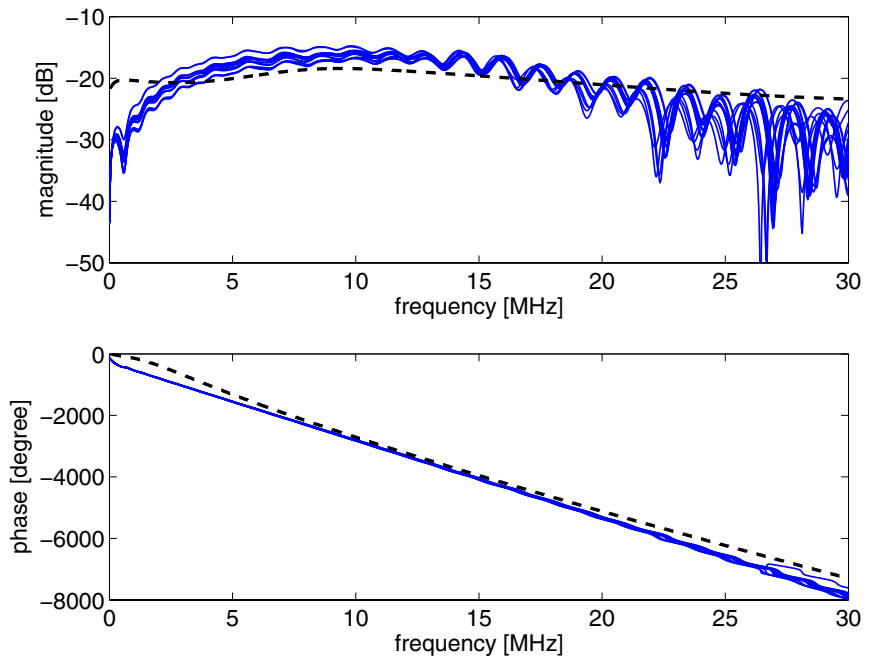

Fig. 7. FEXT coupling function between wire-shield modes whose wires belong to the same twisted pair (Top plot: magnitude. Bottom plot: phase). Solid lines: 20 measured coupling functions. Dashed line: model based on parameters obtained via data fitting.

belong to different pairs. The magnitude levels of FEXT coupling and insertion loss are similar-in certain frequency ranges, the FEXT magnitude exceeds the magnitude of the insertion loss. The match between direct measurements and modelling results is reasonable. The same can be concluded for the extrapolated values except for crosstalk among wires in the same pair where some discrepancy exists in the lowfrequency region. The reason for this mismatch are the poor fitting results of resistance, which has a large impact for low frequencies. For higher frequencies, the impact of inductance and capacitance dominates.

\section{CONCLUSION}

Measurement and modelling results characterizing common-mode propagation in an STP cable were presented. Data fitting using the models from [5] yields acceptable results for inductance and capacitance while the match is poor for resistance. The common-mode FEXT magnitude levels are strong and can exceed the insertion-loss levels, which may be beneficial when exploiting the common mode using MIMO techniques (assuming full cooperation among all modes on both ends of the cable).

\section{ACKNOWLEDGEMENT}

This work was in part supported by the CELTIC project BANITS 2, and by the IP MUSE 2 of the European Union's 6th framework program.

\section{REFERENCES}

[1] C. Valenti, "NEXT and FEXT models for twisted-pair north american loop plant," IEEE Journal on Selected Areas in Communications, vol. 20, no. 5, pp. 893-900, 2002.

[2] T. Magesacher, P. Ödling, P.O. Börjesson, B. Henkel, T. Nordström, R. Zukunft, and S. Haar, "On the capacity of the copper cable channel using the common mode," in Global Telecommunications Conference, 2002. GLOBECOM '02. IEEE, 17-21 Nov. 2002, vol. 2, pp. 1269-1273.
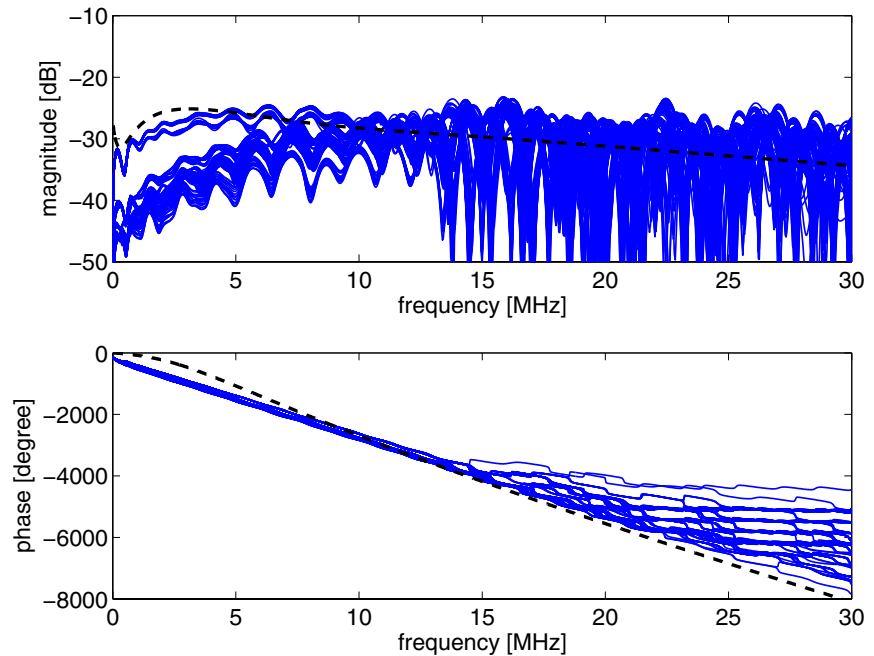

Fig. 8. FEXT coupling function between wire-shield modes whose wires belong to different pairs (Top plot: magnitude. Bottom plot: phase). Solid lines: 360 measured coupling functions. Dashed line: model based on parameters obtained via data fitting.

[3] T. Magesacher, W. Henkel, G. Tauböck, and T. Nordström, "Cable measurements supporting xDSL technologies," Journal e\&i Elektrotechnik und Informationstechnik, vol. 199, no. 2, pp. 37-43, Feb. 2002.

[4] T. Magesacher, P. Ödling, P.O. Börjesson, and T. Nordström, "Verification of multi-pair copper cable model by measurements," IEEE Transactions on Instrumentation and Measurements, vol. 56, no. 5, pp. 1883-1886, Oct. 2007.

[5] ANSI, "Dynamic spectrum management report," Draft Standard T1E1.4/2003-018R, ANSI, Feb. 2004.

[6] J. Cioffi et al., "Vectored DSLs with DSM: The road to ubiquitous gigabit DSLs," in Proc. of the World Telecommunications Congress 2006, (invited paper), Budapest, Hungary, 2006.

[7] G. Ginis and J. M. Cioffi, "Vectored transmission for digital subscriber line systems," IEEE Journal on Selected Areas in Communications, vol. 20, no. 5, pp. 1085-1104, Jun 2002.

[8] C. R. Paul, Analysis of Multiconductor Transmission Lines, ISBN: 978-0471020806. Wiley-Interscience; 1 edition, September 1, 1994.

[9] B. Lee, J. M. Cioffi, S. Jagannathan, K. Seong, Y. Kim, M. Mohseni, and M. H. Brady, "Binder MIMO channel," IEEE Transactions on Communications, vol. 55, no. 8, pp. 1617-1628, August 2007.

[10] B. Lee, J. M. Cioffi, S. Jagannathan, and M. Mohseni, "Gigabit DSL," IEEE Transactions on Communications, vol. 55, no. 9, pp. 1689-1692, September 2007.

[11] C.R. Paul, "Modeling electromagnetic interference properties of printed circuit boards," IBM Journal of Research and Development, vol. 33, no. 1, pp. 33-50, 1989.

[12] International Standard 61156-1, "Multicore and symetrical pair/quad cables for digital communications, part 1: Generic specification," Tech. Rep., IEC, 2002.

[13] T. Mido, H. Ito, and K. Asada, "Test structure for characterizing capacitance matrix of multi-layer interconnects in VLSI," IEIC Transaction on Electronics, Special Issue on Microelectronic Test Structure, vol. E82-C, no. 4, pp. 570-575, 1999. 